CLINICAL STUDY

\title{
Raised serum levels of interleukin-8 and interleukin-18 in relation to bone metabolism in endogenous Cushing's syndrome
}

\author{
Cybèle Kristo $^{1}$, Kristin Godang ${ }^{1}$, Thor Ueland ${ }^{1,3}$, Egil Lien ${ }^{4}$, Pål Aukrust ${ }^{2,3}$, Stig S Frøland ${ }^{2,3}$ and Jens Bollerslev ${ }^{1}$ \\ ${ }^{1}$ Section of Endocrinology, ${ }^{2}$ Section of Clinical Immunology and Infectious Diseases and ${ }^{3}$ Research Institute for Internal Medicine, Medical Department, \\ National University Hospital, N-OO27 Oslo, Norway \\ and ${ }^{4}$ Institute of Cancer Research and Molecular Biology, The Norwegian University of Science and Technology, 7489 Trondheim, Norway
}

(Correspondence should be addressed to C Kristo, Section of Endocrinology, Medical Department, National University Hospital, N-0027 Oslo, Norway; Email: cybele.kristo@rikshospitalet.uio.no)

\begin{abstract}
Objective: It is well known that patients with endogenous Cushing's syndrome (CS) have decreased bone mass and enhanced risk for osteoporotic fractures, secondary to decreased bone formation and increased bone resorption. Immunological mediators, such as cytokines, have recently been shown to influence bone metabolism, and in the present study we examined serum levels of several cytokines, with known or potential effects on bone homeostasis, in 33 consecutive recruited untreated CS patients and 33 age-, sex- and body mass index-matched healthy controls.

Methods: Cytokine levels were measured by enzyme immunoassay and bone mass by dual-energy $\mathrm{X}$-ray absorptiometry.

Results: Our main findings were (i) interleukin (IL)-8 and IL-18 levels were significantly increased in CS patients compared with controls. (ii) Levels of both IL-8 and IL-18 were positively correlated to serum cortisol. (iii) For serum levels of the 'classical' resorptive cytokines, i.e. IL-6 and tumor necrosis factor $\alpha$, no significant differences were found between CS patients and controls. (iv) Raised IL-18 levels were correlated with decreased osteocalcin levels in CS patients.

Conclusions: Our results demonstrated that CS patients have markedly elevated levels of the proinflammatory cytokines IL- 8 and IL-18 in spite of high levels of the immunosuppressive hormone cortisol. These cytokines may be involved in the pathogenesis of disturbed bone homeostasis in CS.
\end{abstract}

European Journal of Endocrinology 146 389-395

\section{Introduction}

Secondary osteoporosis is a major problem in several endocrine and chronic inflammatory disorders, but the pathogenic mechanisms have been only partly elucidated (1-3). Among others, we have recently shown that bone mass as well as bone area are reduced in endogenous Cushing's syndrome (CS), most probably related to decreased bone formation and increased bone resorption $(1,4,5)$. While some of these disturbances in bone metabolism may be a direct consequence of cortisol excess (6), other factors may be involved (e.g. secondary hyperparathyroidism) $(7,8)$, and the mechanisms leading to altered bone homeostasis in CS have not been fully clarified (5).

Systemic and locally produced hormones and growth factors regulate bone metabolism and turnover and recently various cytokines have also been found to be involved in this process $(9,10)$. Several cytokines have bone-resorptive properties in vitro, e.g. interleukin (IL)-1, IL-6 and tumor necrosis factor $\alpha(\mathrm{TNF} \alpha)(11-15)$. Enhanced activity of these cytokines has also been related to postmenopausal (hypogonadtropic) osteoporosis, although the results have been somewhat conflicting (14-18), possibly reflecting the heterogeneity of postmenopausal osteoporosis.

In the present study, we expanded our series of patients with CS (5) and related bone parameters to a panel of 'classical' and 'recently' discovered cytokines and chemokines with known or potential effects on bone homeostasis. In addition to further elucidating the pathogenesis of disturbed bone metabolism in CS, such an approach, examining a patient population with well-described hormonal changes and markedly disturbed bone homeostasis, might also shed light on the relationship between cytokines and bone metabolism in more general terms. 
Table 1 Patient characteristics, bone mineral density and biochemical bone markers in patients with CS and age-, sex and BMI-matched controls. Except for age, BMI and serum cortisol (means \pm S.E.M.) data are given as median and 25th-75th percentiles.

\begin{tabular}{lllr}
\hline & \multicolumn{1}{c}{ CS $(n=33)$} & Controls $(n=33)$ & NS \\
\hline Age & $43 \pm 2$ & $44 \pm 2$ & NS \\
BMI & $28.5 \pm 0.7$ & $27.5 \pm 0.5$ & NS \\
Sex (men/women) (\%) & $27 / 73$ & $24 / 76$ & $<0.001$ \\
Diagnosis (pituitary/adrenal) & $26 / 7$ & $398 \pm 33$ & 0.009 \\
Serum cortisol (nmol/l) & $631 \pm 42$ & $2312(2236,2682)$ & $<0.001$ \\
Bone mineral & & $2828(2589,3265)$ & $<0.001$ \\
$\quad$ Total bone area (CS, $n=23)$ & $2205(1892,2325)$ & $1.19(1.14,1.28)$ & $<0.001$ \\
Total BMC (CS, $n=23)$ & $2335(2035,2585)$ & $1.24(1.10,1.34)$ & $<0.001$ \\
Total BMD (CS, $n=23)$ & $1.10(1.03,1.13)$ & $0.97(0.90,1.12)$ & 0.008 \\
BMD LS (CS, $n=32)$ & $1.02(0.99,1.13)$ & $0.34(0.32,0.38)$ & 0.025 \\
BMD FN (CS, $n=32)$ & $0.86(0.74,0.91)$ & $0.75(0.71,0.82)$ & $\mathrm{NS}$ \\
BMD UD (CS, $n=26)$ & $0.31(0.28,0.35)$ & $3.7(2.7,4.9)$ & 0.001 \\
BMD 33\% (CS, $n=26)$ & $0.70(0.65,0.79)$ & $2848(2308,3464)$ & \\
Bone markers & & & \\
Osteocalcin & $3.1(1.8,4.7)$ & & \\
CTX-1 & $4009(2920,4956)$ & & \\
\hline
\end{tabular}

LS (lumbal columna); FN (femoral neck); UD (ultra distal radius/ulna); TR (truncus).

NS, not significant.

\section{Patients and methods}

\section{Patients and controls}

Thirty-three consecutive recruited patients with endogenous CS (Table 1) (mean age $43 \pm 2$ years, body mass index (BMI) $28.5 \pm 0.7 \mathrm{~kg} / \mathrm{m}^{2}$ ) were systematically evaluated before treatment and compared with 33 age-, sex- and BMI-matched healthy controls (mean age $44 \pm 2$ years, BMI $27.5 \pm 0.5 \mathrm{~kg} / \mathrm{m}^{2}$ ). The patients had a typical history and objective findings of CS. The diagnosis was confirmed by established abnormal diurnal rhythm of serum cortisol, resistance to a conventional 2-day dexamethasone suppression test and elevated 24-h urine free cortisol levels. Hypogonadism in men was defined as androgen index $<25 \%$ (normal range 25-200\%) and in women as secondary amenorrhea for at least 6 months. The study was approved by the local ethical committee and conducted according to the Declaration of Helsinki II. Informed consent was obtained from all patients.

\section{Osteodensitometric measurements}

Bone mineral density (BMD), bone mineral content (BMC) and area were measured in the lumbar spine (L2-L4, anterior-posterior), the left femoral neck and non-dominant forearm, using dual-energy X-ray absorptiometry (DEXA; Lunar DPX-L, software version 1.31; Lunar Corporation, WI, USA), as previously described (5). BMC is expressed in $\mathrm{g}$ and BMD is expressed in $\mathrm{g} / \mathrm{cm}^{2}$. For each bone region measured, the area in $\mathrm{cm}^{2}$ was determined. The software uses a 'threshold' technique to determine if a data point is air, tissue or bone. The cuts define a region, separating out data points into each of the defined locations. The software adds up the bone data points for a region and calculates the area based on the sample size of the bone. In addition, total body determination of BMC and BMD was evaluated in 23 of the patients and all 33 age- and BMI-matched controls (5).

\section{Biochemical measurements}

Blood samples were drawn after an overnight fast into pyrogen-free vacuum blood collection tubes without any additives (Becton Dickenson, San Diego, CA, USA). Tubes were immediately immersed in melting ice, allowed to clot for $2 \mathrm{~h}$ and centrifuged for $15 \mathrm{~min}$ $\left(1000 \mathrm{~g}\right.$ at $4{ }^{\circ} \mathrm{C}$ ) before storing serum at $-80^{\circ} \mathrm{C}$ in multiple aliquots until analyzed. Samples were thawed only once.

Analyses of bone-related markers and hormones in serum The bone formation marker osteocalcin was measured by immunoradiometric assay with a commercial kit from Incstar Corporation, Stillwater, MI, USA. The assay measures intact osteocalcin 1-49. Degradation products of the C-terminal telopeptides of type I collagen (CTX-1) were measured in serum with a commercial enzyme immunoassay (EIA; Crosslaps) (19) from Osteometer Bio-Tech A/S, Herlev, Denmark. Serum and free cortisol in 24-h urine samples was measured by radioimmunoassay, using commercial kits from Orion Diagnostics, Espoo, Finland.

Cytokine measurement in serum $\mathrm{TNF} \alpha$ (detection limit $3 \mathrm{pg} / \mathrm{ml}$ ) and IL-10 (detection limit $0.5 \mathrm{pg} / \mathrm{ml}$ ) were quantified by EIA (BioSource Europe, Nivelles, Belgium) as described (20). IL-1 $\beta$ (detection limit $0.1 \mathrm{pg} / \mathrm{ml}$ ), IL-1 receptor antagonist (IL-1Ra; 
detection limit $22.0 \mathrm{pg} / \mathrm{ml}$ ), IL-6 (detection limit $0.094 \mathrm{pg} / \mathrm{ml}$ ), IL-8 (detection limit $2 \mathrm{pg} / \mathrm{ml}$ ), IL-18 (detection limit $15 \mathrm{pg} / \mathrm{ml}$ ), and monocyte chemotactic protein (MCP-1) (detection limit $8 \mathrm{pg} / \mathrm{ml}$ ) were measured by EIAs as recommended by the manufacturer (R\&D Systems, Minneapolis, MN, USA). Serum levels of soluble TNF receptors (sTNFRs), p55 and p75, were analyzed by EIAs (detection limit between $150-300 \mathrm{pg} / \mathrm{ml}$ ) as described by Liabakk et al. (21) Intra- and interassay coefficients of variation were $<10 \%$ for all assays.

\section{Statistical analysis}

Mann-Whitney rank sum test for unpaired data was used for comparisons between variables. Relationships between variables were tested using Spearman's rank correlation test. Throughout, we report two-tailed $P$ values, and values $<0.05$ were considered significant. However, particular attention should be directed towards smaller $P$ values (i.e. $<0.01$ ) because a considerable number of $P$ values have been calculated. Percentual differences between groups in the text are based on medians.

\section{Results}

\section{Osteodensitometry}

As previously reported (5), total bone area, total BMC and total BMD were significantly reduced in patients with CS compared with controls $(5 \%, 17 \%$ and $8 \%$ respectively, $P<0.001)$. Further, CS patients had significantly decreased $\mathrm{BMD}$ in the lumbar spine (18\%, $P<0.001)$, femoral neck (11\%, $P<0.001)$, ultra distal radius $(9 \%, P=0.01)$ and radius $33 \%$ $(7 \%, P<0.03)$ (Table 1). Similar results were observed for $\mathrm{BMC}$ in these regions (data not shown). No men were found to be hypogonodal, whereas 18 women had secondary amenorrhea. These women had significantly higher frequency of osteopenia/osteoporosis (osteopenia defined as a T-score -0.5 to 2.5 and osteoporosis as a T-score $<-2.5$ measured by DEXA) in femoral neck $(P=0.0364$; data not shown $)$.

\section{Serum levels of bone-related markers}

Serum levels of osteocalcin were not significantly decreased $(21 \%, P=0.11)$, while CTX-1 was significantly increased $(47 \%, P<0.005)$ in $\mathrm{CS}$ patients compared with controls (Table 1).

\section{Serum levels of cytokines}

When examining serum cytokine levels in CS patients and healthy controls, several significant findings were revealed (Fig. 1). First, CS patients had markedly increased levels of the proinflammatory cytokine
IL-18 $(67 \%, P<0.05)$ and in particular of the CXC chemokine IL-8 $(215 \%, P<0.001)$, compared with healthy controls. Secondly, while serum levels of $\mathrm{TNF} \alpha$ did not differ between CS patients and controls, the patients had decreased levels of both types of sTNFRs although the differences in soluble p55 TNFR did not reach statistical significance (p75: 17\%, $P<0.05$; p55: 16\%, $P=0.06$ ). Finally, investigating the components of the IL-1 system, we found that while CS patients had slightly, but significantly decreased IL-1 $\beta$ levels $(44 \%, P<0.05)$, IL-1Ra was significantly elevated $(27 \%, P<0.05)$. No differences were found between the CS patients and controls with respect to IL-6, IL-10 or MCP-1 levels. Similar patterns of cytokine levels were found in both adrenal $(n=7)$ and pituitary $(n=26)$-dependent CS (data not shown).

\section{Relationships between cytokine levels, bone-related markers and BMD}

In CS patients, but not in controls, the increased IL-8 and IL-18 levels were significantly positively correlated with serum cortisol (Fig. 2). Moreover, in CS patients, but not in healthy controls, IL-18 levels were significantly negatively correlated with osteocalcin $(r=-0.48, \quad P<0.01)$. No significant correlations were found between cytokine levels and CTX-1 in patients or controls. When correlating these cytokines with BMD, no significant correlations were found in CS patients while IL-8 was negatively correlated to total $\mathrm{BMD}(r=-0.42, P<0.05)$, lumbar spine $(r=-0.44, P<0.05)$ and ultra distal radius $(r=-0.54, P<0.001)$ in controls.

\section{Discussion}

To some degree most patients with CS have hypogonadotropic hypogonadism, either directly because of the pituitary adenoma in Cushing's disease, or indirectly related to inhibition of gonadotropins by glucocorticoid (6). As expected, the women with hypogonadism had significantly decreased bone mass in the femoral neck compared with the eugonodal female patients. Compelling and consistent evidence indicates that sex steroids directly regulate the IL-6 gene. Estrogens inhibit IL- 6 and loss of this sex steroid may cause an upregulation of IL- 6 production which, in turn, may result in enhanced bone resorption (14). However, in the present study, CS patients had IL-6 levels within normal limits. Furthermore, although IL- $1 \beta$ and TNF $\alpha$ have been suggested to induce bone disturbances in various disorders $(2,22)$, we found no elevation in serum levels of these 'classical' resorptive cytokines in CS patients. However, although IL-1 $\beta$ was not raised in CS patients, those with high IL-1 $\beta$ levels had decreased BMD. Moreover, and most importantly, serum levels may not necessarily reflect the 

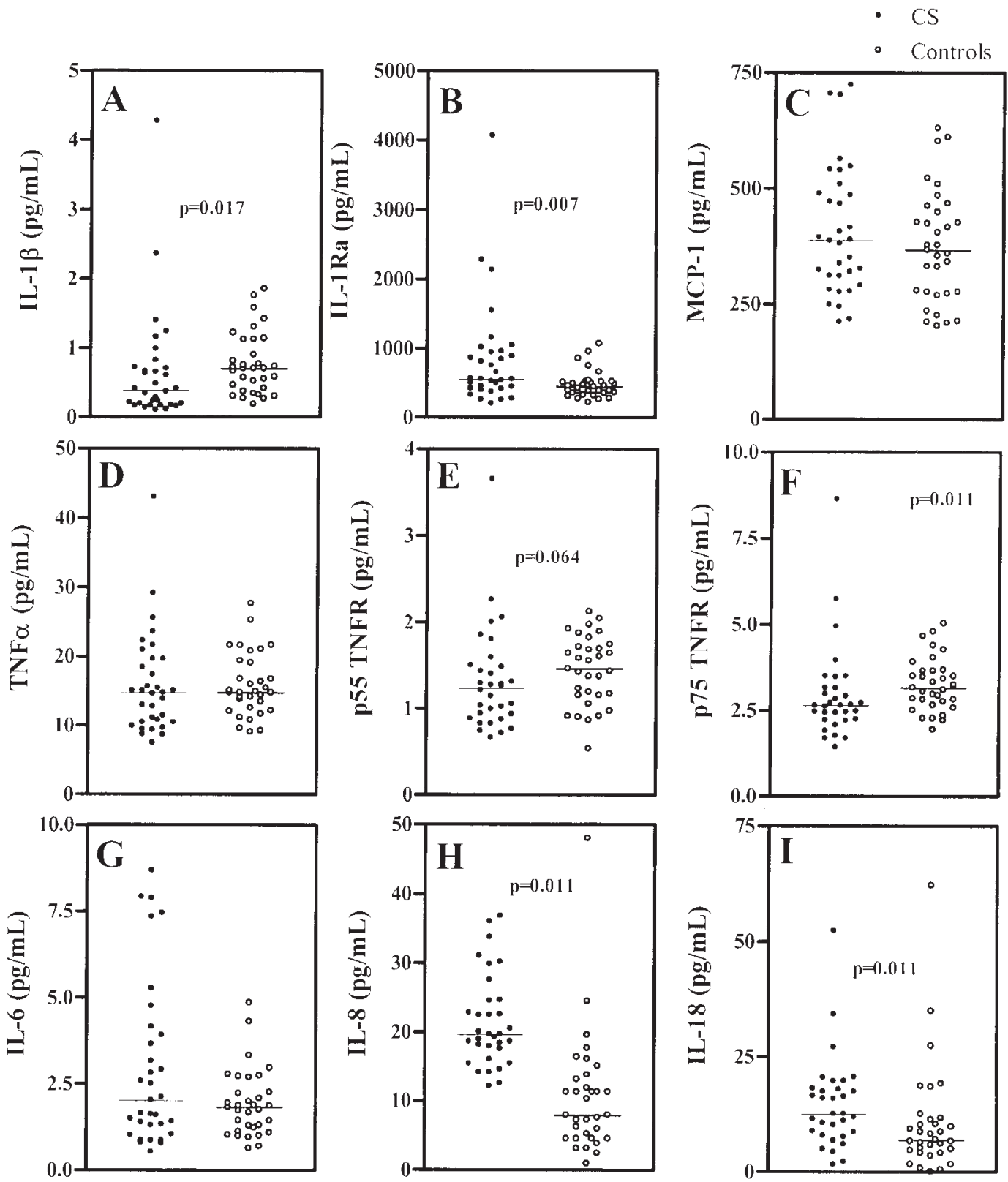

Figure 1 Serum levels of (A) IL-1 $\beta$, (B) IL-1Ra, (C) MCP-1, (D) TNF $\alpha$, (E) p55 TNFR, (F) p75 TNFR, (G) IL-6, (H) IL-8 and (I) IL-18 in 33 patients with CS $(\bullet)$ and 33 age-, sex- and BMI-matched healthy controls $(O)$.

cytokine levels in the bone microenvironment and our results do not exclude a role for IL-1 $\beta$ or other "classical' resorptive cytokines in the pathogenesis of disturbed bone metabolism in CS.

Major findings in the present study were the elevated levels of the proinflammatory cytokines IL- 8 and IL-18 in CS patients, significantly correlated with cortisol levels. Moreover, this elevation of IL-18 was associated with decreased osteocalcin levels. In patients with elevated levels of the immunosuppressive hormone cortisol these findings may seem surprising. In fact, cortisol administration in vivo has been associated with enhanced levels of the anti-inflammatory cytokine IL-10 and decreased levels of proinflammatory cytokines (23). However, it was recently shown in animal models that acute stress, through adrenocorticotropic hormone, stimulates the expression of IL-18 in glucocorticoid-producing cells of the adrenal cortex. Furthermore, this production was not inhibited by corticosterone, suggesting an immunostimulatory role for IL-18 during acute stress (24). Nonetheless, the effect of cortisol on IL-18 levels has not been investigated, and the outcome of short-time in vivo cortisol administration may not necessarily reflect the situation 

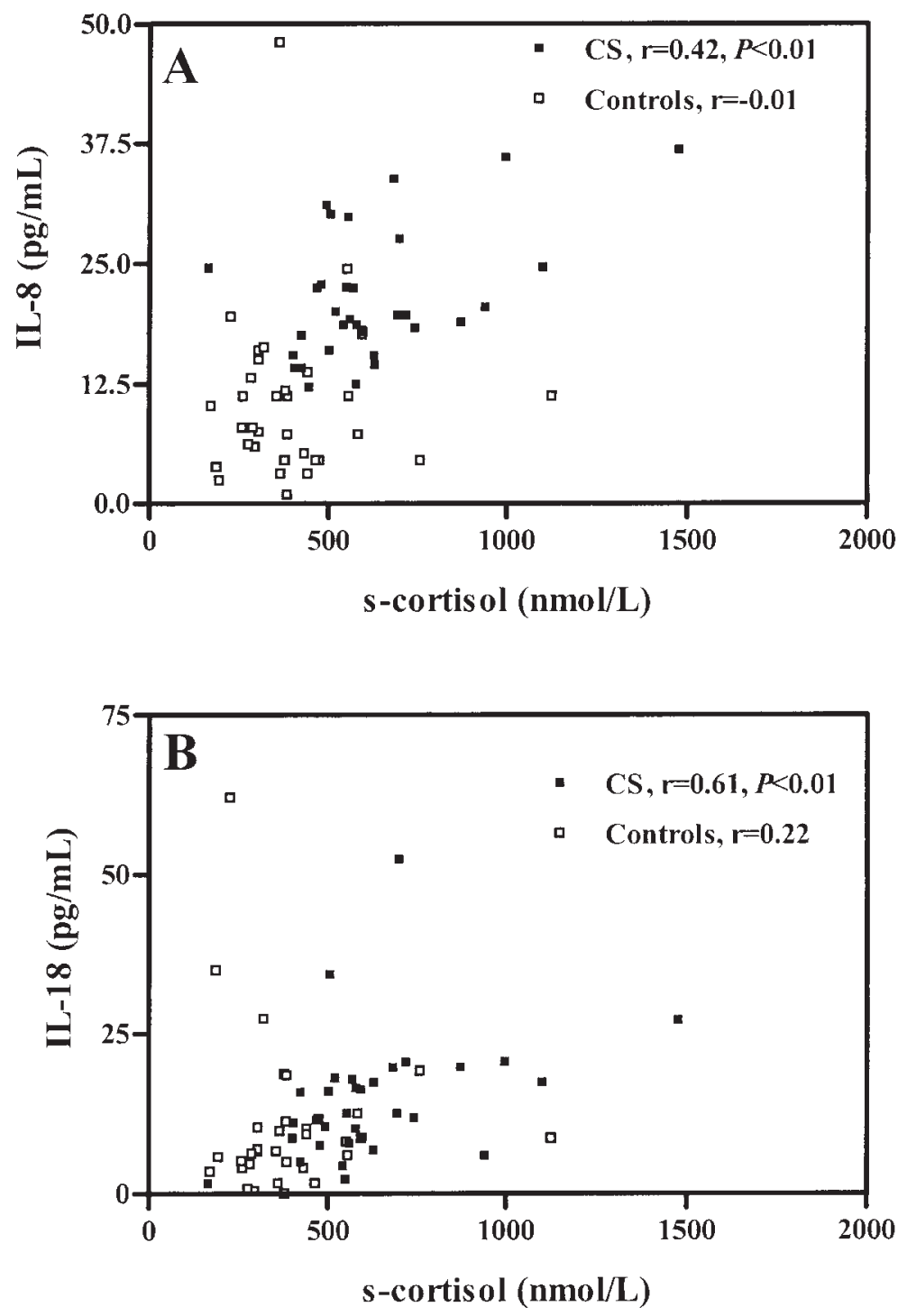

Figure 2 Correlations between serum levels of cortisol (s-cortisol) and (A) IL-8 and (B) IL-18 in 33 patients with CS and 33 age-, sex- and BMI-matched healthy controls.

in CS patients with persistently elevated cortisol levels over several months or years. Although the biological significance of these correlations between high levels of cortisol and IL-8 and IL-18 is at present unclear, it may possibly suggest that some immunopathogenic mechanisms may be operating in CS $(24,25)$.

Several cytokines are known to influence bone homeostasis by increasing bone resorption (e.g. IL-6 and $\mathrm{TNF} \alpha)(11,12,26)$, but few studies have addressed the role of IL- 8 and IL-18 in this process $(26,27)$. However, both IL-8 and IL-18 can be produced by osteoblasts $(28,29)$ and IL-8 by osteoclasts in response to proinflammatory stimuli (2). Thus a local rise in IL-8 levels could potentially contribute to regional bone loss through suppressive effects on osteoblasts and recruitment of osteoclasts to new sites of resorption. However, the responses to inflammatory stimuli are suppressed by glucocorticoids in these cells, as well as other cells that are potential sources to systemic IL-8 levels. Thus mechanisms other than those demonstrated in vitro may contribute to the elevated IL-8 levels found in CS in this study. Moreover, IL-8 may also indirectly contribute to disturbed bone homeostasis in various inflammatory disorders by modulating parathyroid hormone secretion (30). IL-18, originally identified as interferon $\gamma$-inducing factor, is related to the IL-1 family in terms of its structure, processing, receptor, signal transduction pathway and proinflammatory properties $(25,27)$ but, in contrast to the resorptive abilities of IL-1 $\beta$, the effects of IL-18 seem to be bone protective; first by inhibiting osteoclast formation through effects on 
granulocyte macrophage colony-stimulating factor (29), but also by upregulating osteoblastic production of osteoprotegerin (31). Nevertheless, few studies have been performed and the exact role of IL- 8 and IL-18 in bone homeostasis will have to be investigated further.

In conclusion, although CS patients have increased levels of the anti-inflammatory and immunosuppressive hormone cortisol, the present study demonstrates that these patients also have elevated serum levels of the proinflammatory cytokine IL-18 and the CXC chemokine, IL-8. As for IL-18, this rise in cytokine level was significantly correlated with decreased osteocalcin levels in these patients. Although serum levels might not necessarily reflect the cytokine levels in the bone microenvironment, our findings may suggest that some immunopathogenic mechanisms may be operating in CS, particularly in the induction of disturbed bone homeostasis.

\section{References}

1 Manning PJ, Evans MC \& Reid IR. Normal bone mineral density following cure of Cushing's syndrome. Clinical Endocrinology $199236229-234$.

2 Rothe L, Collin-Osdoby P, Chen Y, Sunyer T, Chaudhary L, Tsay A et al. Human osteoclasts and osteoclast-like cells synthesize and release high basal and inflammatory stimulated levels of the potent chemokine interleukin-8. Endocrinology $1998 \quad 139$ 4353-4363.

3 Harper KD \& Weber TJ. Secondary osteoporosis. Diagnostic considerations. Endocrinology and Metabolism Clinics of North America $199827325-348$.

4 Chiodini I, Carnevale V, Torlontano M, Fusilli S, Guglielmi G, Pileri $\mathrm{M}$ et al. Alterations of bone turnover and bone mass at different skeletal sites due to pure glucocorticoid excess: study in eumenorrheic patients with Cushing's syndrome. Journal of Clinical Endocrinology and Metabolism 1998 $831863-1867$.

5 Godang K, Ueland T \& Bollerslev J. Decreased bone area, bone mineral content, formative markers, and increased bone resorptive markers in endogenous Cushing's syndrome. European Journal of Endocrinology 1999141 126-131.

6 Canalis E. Clinical review 83: Mechanisms of glucocorticoid action in bone: implications to glucocorticoid-induced osteoporosis. Journal of Clinical Endocrinology and Metabolism 1996 $813441-3447$.

7 Boonen S, Broos P, Verbeke G, Aerssens J, Van Herck E, Jans I et al. Calciotropic hormones and markers of bone remodeling in agerelated (type II) femoral neck osteoporosis: alterations consistent with secondary hyperparathyroidism-induced bone resorption. Journal of Gerontology Series A, Biological Sciences and Medical Sciences 199752 286-293.

8 Masiukiewicz US \& Insogna KL. The role of parathyroid hormone in the pathogenesis, prevention and treatment of postmenopausal osteoporosis. Aging $199810232-239$.

9 Ueland T, Bollerslev J, Godang K, Muller F, Frøland SS \& Aukrust P. Increased levels of biochemical markers of bone turnover in relation to persistent immune activation in common variable immunodeficiency. European Journal of Clinical Investigation 2000 $301-9$.

10 Price JS, Oyajobi BO \& Russell RG. The cell biology of bone growth. European Journal of Clinical Nutrition $1994 \mathbf{4 8}$ (Suppl 1) S131-S149.
11 Mundy GR. Cytokines and growth factors in the regulation of bone remodeling. Journal of Bone and Mineral Research $1993 \mathbf{8}$ S505-S510.

12 Ishimi Y, Miyaura C, Jin C, Akatsu T, Abe E, Nakamura Y et al. IL-6 is produced by osteoblasts and induces bone resorption. Journal of Immunology 1990145 3297-3303.

13 Passeri G, Girasole G, Manolagas SC \& Jilka RL. Enodogenous production of tumor necrosis factor by primary cultures of murine calvarial cells: influence on IL-6 production and osteoclast development. Journal of Bone and Mineral Research 1994 24 109-126.

14 Manolagas SC. Role of cytokines in bone resorption. Bone 1995 17 63S-67S.

15 Rifas L. Bone and cytokines: beyond IL-1, IL-6 and TNF-alpha. Calcified Tissue International 199964 1-7.

16 McKane WR, Khosla S, Peterson JM, Egan K \& Riggs RL. Circulating levels of cytokines that modulate bone resorption: effects of age and menopause in women. Journal of Bone and Mineral Research 19949 1313-1318.

17 Pacifici R. Estrogen, cytokines, and pathogenesis of postmenopausal osteoporosis. Journal of Bone and Mineral Research 1996 11 1043-1051.

18 Khosla S, Peterson JM, Egan K, Jones JD \& Riggs BL. Circulating cytokine levels in osteoporotic and normal women. Journal of Clinical Endocrinology and Metabolism $1994 \mathbf{7 9}$ $707-711$.

19 Bonde M, Garnero P, Fledelius C, Qvist P, Delmas PD \& Christiansen C. Measurement of bone degradation products in serum using antibodies reactive with an isomerized form of an 8 amino acid sequence of the C-telopeptide of type I collagen. Journal of Bone and Mineral Research $1997 \mathbf{1 2}$ 1028-1034.

20 Aukrust P, Lien E, Kristoffersen AK, Muller F, Haug CJ, Espevik T et al. Persistent activation of the tumor necrosis factor system in a subgroup of patients with common variable immunodeficiency possible immunologic and clinical consequences. Blood $1996 \mathbf{8 7}$ 674-681.

21 Liabakk NB, Sundan A, Lien E, Laegreid A, Bombara MP, Ranges $\mathrm{G}$ et al. The release of soluble p55 TNF receptor from U937 cells studied by a new p55 immunoassay. Journal of Immunological Methods 1993163 145-154.

22 Aukrust P, Haug CJ, Ueland T, Lien E, Muller F, Espevik T et al. Decreased bone formative and enhanced resorptive markers in human immunodeficiency virus infection: indication of normalization of the bone-remodeling process during highly active antiretroviral therapy. Journal of Clinical Endocrinology and Metabolism $199984145-150$.

23 Dandona P, Aljada A, Garg R \& Mohanty P. Increase in plasma interleukin-10 following hydrocortisone injection. Journal of Clinical Endocrinology and Metabolism 199984 1141-1144.

24 Conti B, Sugama S, Kim Y, Tinti C, Kim H, Baker H et al. Modulation of IL-18 production in the adrenal cortex following acute ACTH or chronic corticosterone treatment. Neuroimmunomodulation $200081-7$.

25 Dinarello CA. IL-18: a TH1-inducing, proinflammatory cytokine and new member of the IL-1 family. Journal of Allergy and Clinical Immunology 1999103 11-24.

26 Martin TJ, Romas E \& Gillespie MT. Interleukins in the control of osteoclast differentiation. Critical Reviews in Eukaryotic Gene Expression $1998 \mathbf{8} 107-123$.

27 Gillespie MT \& Horwood NJ. Interleukin-18: perspectives on the newest interleukin. Cytokine and Growth Factor Reviews 19989 $109-116$.

28 Udagawa N, Horwood NJ, Elliott J, Mackay A, Owens J, Okamura $\mathrm{H}$ et al. Interleukin-18 (interferon-gamma-inducing factor) is produced by osteoblasts and acts via granulocyte/macrophage colony-stimulating factor and not via interferon-gamma to inhibit osteoclast formation. Journal of Experimental Medicine $1997 \mathbf{1 8 5}$ 1005-1012.

29 Walsh CA, Birch MA, Fraser WD, Ginty AF \& Gallagher JA. Cytokine expression by cultured osteoblasts from patients with 
osteoporotic fractures. International Journal of Experimental Pathology 20008 159-163.

30 Angeletti RH, D’Amico T, Ashok S \& Russell J. The chemokine interleukin-8 regulates parathyroid secretion. Journal of Bone and Mineral Research 199813 1232-1237.

31 Makiishi-Shimobayashi C, Tsujimura T, Iwasaki T, Yamada N, Sugihara A, Okamura $\mathrm{H}$ et al. Interleukin-18 up-regulates osteoprotegerin expression in stromal/osteoblastic cells Biochemical and Biophysical Research Communications 2001281 $361-366$.

Received 23 May 2001

Accepted 9 November 2001 